\title{
Investigation of Uniaxial Asymmetric High-cycle Fatigue Failure Behavior of 7075-T651 Aluminum Alloy Sheet
}

\author{
Da-Lian YANG ${ }^{1, a}$, Yi-Lun LIU ${ }^{1,2, b^{*}}$, Song-Bai LI ${ }^{1, c}$, Jiu-Huo $\mathrm{YI}^{1, d}$, Jie TAO ${ }^{1, \mathrm{e}}$ \\ ${ }^{1}$ State Key Laboratory for High Performance and Complex Manufacturing, School of Mechanical and \\ Electrical Engineering, Central South University, Changsha 410083, China \\ ${ }^{2}$ Light Alloy Research Institute, Central South University, Changsha 410083, China

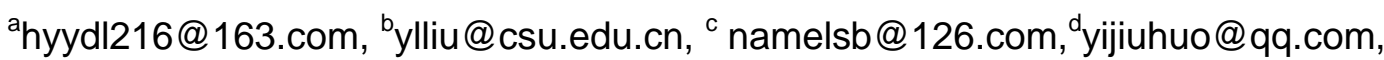 \\ ecaroltaojie@126.com \\ ${ }^{*}$ Corresponding author
}

Keywords: 7075 Aluminum Alloy, High Cycle Fatigue (HCF), S-N Curve, Fatigue Fracture.

\begin{abstract}
In this investigation, the high cycle fatigue life of 7075-T651 aluminum sheets under different stress levels were tested, and S-N curves were obtained by fitting the test data. For the problem that the traditional S-N curve fitting methods without considering the effect of fatigue limit on the S-N curve fitting accuracy, the traditional fitting methods were improved, the results show that the improved method can reduce the fitting error. The high- cycle fatigue fracture morphology of $260 \mathrm{MPa}$ was observed by scanning electron microscopy (SEM). The observations reveal that the high cycle fatigue fracture morphology shows ductile fracture features. The main reason of the crack nucleation is that the second phase particles on the sub-surface, but the small dispersed precipitates can improve the fatigue performances. The morphology of the fatigue crack propagation is "stair" distribution. The fatigue crack transient beak region is characterized by the mixed-rupture characteristics of quasi-cleavage and dimples.
\end{abstract}

\section{Introduction}

With the advantages of small density, high strength, good machinability and heat treatment performances, the aluminum alloy of 7075 are widely used in the fields of aerospace industry, transportation, electronic communication [1-2]. These components suffered from the external alternating loads inevitably in service, eventually, the materials of components appear the irreversible fatigue failure, and either affect the normal operation of the equipment, or causes a serious disaster. Therefore, it is very significance to further investigate the fatigue performances and the fatigue mechanism of the 7075 aluminum alloy so that it can provide a guide for the structural design to reduce the fatigue accidents.

In the past decade, many domestic and foreign scholars have done a lot of work on the fatigue performance investigation of aluminum alloy and have obtained a series of achievements. E. Cirik [3] investigated the effect of anodic oxidation on the fatigue performance of 7075-T6 alloy and fatigue tests with pre-corroded specimens showed that fatigue life of coated specimens was significantly affected by pre-corrosion, except for the specimen with the thickest coating layer. T. W. Zhao [4] tested the fatigue crack propagation rate of 7075-T651 aluminum alloy with the standard and non-standard compact specimens under the conditions of different stress ratio (R), overload, underload, high-low order, by improving the model of Wheeler [5], a good prediction results that the loading condition influence on the fatigue crack growth were obtained. R. S. Sachin [6] revealed the fretting fatigue behavior in 7075-T6 aluminum alloy use constant amplitude cyclic loading, using a Zeiss confocal microscope and a Hitachi Scanning Electron Microscope (SEM) to characterize the fretting fatigue damage, the results showed that fretting degradation depends on the microstructure of the material. J. Han [7] discovered the fatigue mechanism and fracture characteristics of the 7075-T651 aluminum alloy use the standard cylinder specimen, meanwhile, he obtained the fatigue life and S-N curve under different stress amplitudes.

Despite comprehensive work, many questions remain to be answered, the fatigue mechanism of 7075-T651 aluminum alloy based on the low cycle fatigue (LCF) testing and use the cylinder 
specimens usually, and there are less investigation of the fatigue performance of 7075-T651, especially, the high-cycle fatigue $(\mathrm{HCF})$ performance should be studied more in-depth. In the current investigation, the fatigue performance often based on the medium thick sheet, but the fatigue performance of the aluminum alloy sheet was less studied. In this investigation, high-cycle fatigue experiments will be conducted using 7075-T651 aluminum alloy sheet under the conditions of different stress amplitude load, so that it can provide a fatigue performance guide for the application of 7075-T651 aluminum alloy.

\section{Experimental}

\section{Material and Specimens}

The material used in the current experimental investigation was 7075-T651 aluminum alloy sheets which come from Europe (4mm thick). The chemical composition as shown in Table 1.

Tab. 1 Chemical composition of aluminum alloy 7075-T651 (wt. \%)

\begin{tabular}{ccccccccc}
\hline $\mathrm{Ti}$ & $\mathrm{Mn}$ & $\mathrm{Si}$ & $\mathrm{Fe}$ & $\mathrm{Cr}$ & $\mathrm{Cu}$ & $\mathrm{Mg}$ & $\mathrm{Zn}$ & $\mathrm{Al}$ \\
\hline 0.019 & 0.034 & 0.06 & 0.17 & 0.18 & 1.5 & 2.45 & 5.71 & $\mathrm{Bal}$. \\
\hline
\end{tabular}

7075 aluminum alloy, in temper T651, is a kind of high strength aluminum alloy, widely used in the aerospace industry, the ultimate tensile strength of longitude- trans-verse orientation (L-T) up to $587 \mathrm{MPa}(85.82 \mathrm{ksi})$, the $0.2 \%$ offset yield strength of longitude- transverse orientation (L-T) up to $519 \mathrm{MPa}(75.88 \mathrm{ksi})$ and the elongation is $13.0 \%$. The 7075-T651 aluminum alloy sheets are detected by X-ray detector and there are no obvious defects. The microstructure as shown in Fig. 1.

In this investigation, the uniaxial arc shaped sheet specimens, conforming to the specifications in ASTM, were machined from a 7075- T651 aluminum alloy sheet, $4 \mathrm{~mm}(0.157 \mathrm{in}$.) thick, The stress axes of the specimens were parallel (longitudinal) and perpendicular (transverse) to the rolling direction, as shown in Fig. 2. The cutting method used the wire cutting technology in order to reduce damage. Polished the machined surface of specimen with fine sandpaper uniformly in order to minimize the stress concentration.

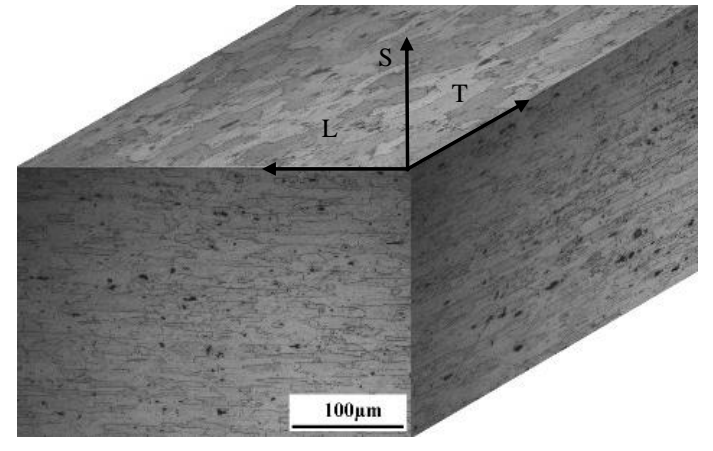

Fig. 1 The microstructure of 7075-T651 aluminum alloy.

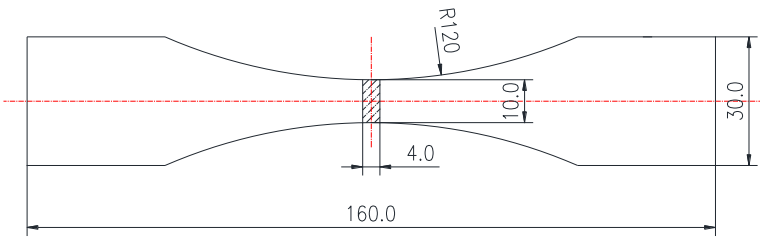

Fig. 2 The shape and the size of high cycle fatigue specimen (unit: $\mathrm{mm}$ )

\section{HCF Testing}

Cyclic- stress- controlled high-cycle fatigue tests were performed on a fully-automated, closed-loop servo hydraulic materials test system which was made by the United States MTS company. This test system with a load cell which can provide the maximum static load $50 \mathrm{kN}$ and the maximum dynamic load $30 \mathrm{kN}$. The testing conforming to the specifications in ASTM. The stress-controlled tests were conducted at room temperature $\left(25^{\circ} \mathrm{C}\right)$, in the air, at a constant cyclic frequency of $40 \mathrm{~Hz}$ and a stress ratio $R=0.1$, the loading force changed according to the sine curve and the maximum stress levels were $350 \mathrm{MPa} \sim 140 \mathrm{MPa}$. The whole test process was completed automatically and the datum was recorded.

Twenty-one specimens were tested. Stopping the load if the cycles more than $10^{7}$ because if the cycles more than $10^{7}$, it is generally considered that the specimen survives forever under the stress 
level. The fatigue fracture morphology observation was performed on the scanning electron microscope (TESCAN MORA3 LMU), the accelerating voltage was $20 \mathrm{KV}$, and the energy dispersive spectrometry was conducted on the OXFORD instrument.

\section{S-N Curve Fitting and Fatigue Limit Estimation}

Usually, to ensure the fitting S-N curve can accurately describe the fatigue life properties of material, a large amount of test datum is required, however, it is great expensive to obtain a large amount of experimental datum in practice. Using the up and down method can obtain the fatigue limit of material, but the higher the test accuracy desired, the greater the amount of tests required and the higher the costs increased. If the fatigue limit is unknown, the fitting results of the traditional S-N fitting method are different from the test data, especially, for higher cycle life, so, the fatigue limit estimate by the S-N curve is different from the real value. To solve these problems, the traditional S-N curve fitting method was improved, before fitting S-N curve, the fatigue limit was pre-estimated. The details as follows:

For $l$ stress levels $S_{i}>S_{2}>\cdots>S_{l}$, if the specimen failure under the stress level $S_{i}$ and the cycle number $N_{i}<10^{7}$, but not failure under the stress level $S_{i+1}$ and the cycle number $N_{i+1}>10^{7}$, then it can be estimated that the fatigue limit in the range of $S_{i}$ to $S_{i+1}$, and the test data $\left\{S_{i+1}, N_{i+1}\right\}$ cannot participate $\mathrm{SN}$ curve fitting. Calculating:

$$
\hat{S}_{i+1}=\left(S_{i}+S_{i+1}\right) / 2
$$

where $\hat{S}_{i+1}$ is the pre-estimate fatigue limit and the cycle number $\hat{N}_{i+1}=10^{7}$.

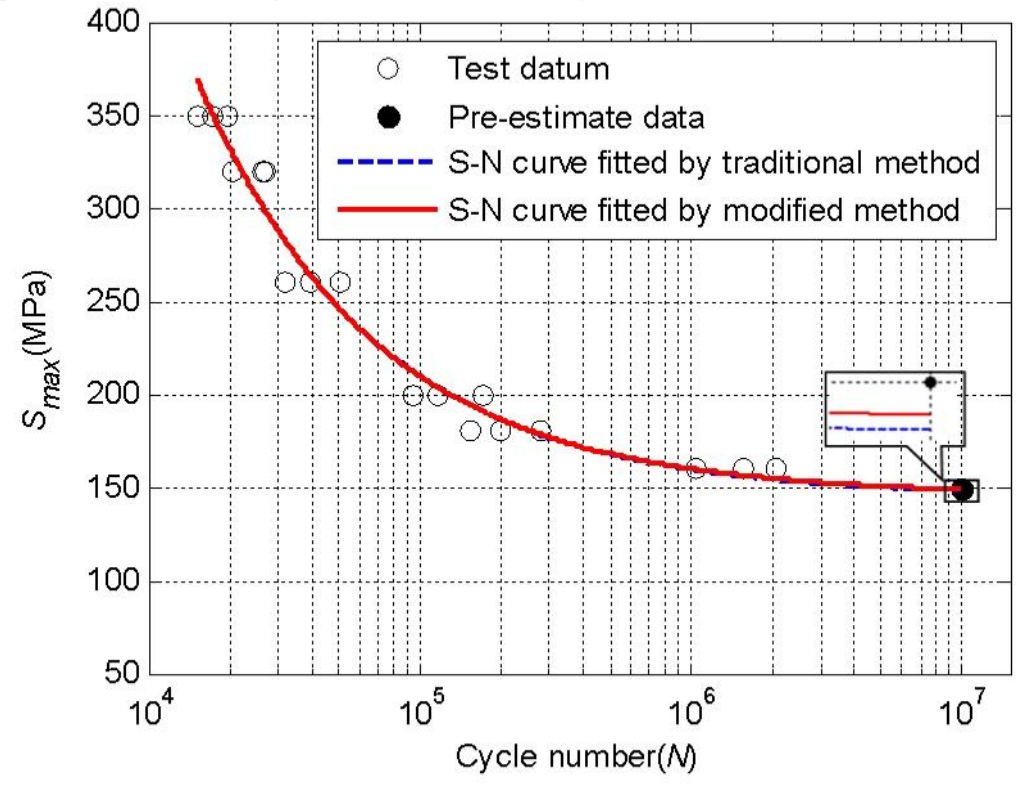

Fig.3 S-N curve of 7075-T651 aluminum alloy

To consider the impact of the fatigue limit on the S-N curve, add the data $\left\{S_{i+1}, N_{i+1}\right\}$ to the test data set. In this testing, the specimens no fractured under the condition of $160 \mathrm{MPa}$ and the cycle number less than $10^{7}$, but fractured under the condition of $140 \mathrm{MPa}$ and the cycle number greater than $10^{7}$, so, before fitting S-N curve, add the data $\left\{150,10^{7}\right\}$. Finally, the fatigue limit can be obtained from the S-N curve by calculating the cycle number is equal to $10^{7}$, this process is the second estimation.

The S-N curve of 7075-T651 aluminum alloy as show in Fig. 3. Seen from Fig. 3, the S-N curve of 7075 aluminum alloy without horizontal section, test datum are distributed on both sides of curve evenly, which demonstrates that the test is valid. 
It also can be seen from Fig.3 that the fatigue limit, which are estimated by S-N curve use the modified data, are higher than use the test datum, and the fatigue limit, which are estimated use the modified datum, is closer to the real fatigue limit which in the range of $140 \mathrm{MPa}$ to $160 \mathrm{MPa}$. All results show that the $\mathrm{S}-\mathrm{N}$ curves fitted use the modified datum can describe the fatigue life of material more accurate. Thereby, it can be estimated the fatigue limit is $149.0033 \mathrm{MPa}$, which less slightly than the pre-estimate fatigue limit.

\section{Fatigue Fracture Morphology Observation}

\section{From the Macroscopic Scale}

The fatigue fracture morphology of stress level $260 \mathrm{MPa}$ was observed by SEM. The low magnification SEM photographs are shown in Fig.4. Seen from the Fig.4 (a), there are three typical regions: the fatigue crack initiation region (A), the fatigue crack propagation region $(\mathrm{B})$ and the fatigue transient break region (C), among them, the area of the fatigue transient break region is largest.

The Ref [7] pointed out that for the specimens without internal tissue defects, the fatigue crack often originated from sub-surface, in this investigation, the fatigue crack initiated from subsurface too.

The main incentive of the fatigue crack initiation is that the resistance of surface is smaller and is easier to form the local stress concentration, the material appear local plastic deformation under the local high stress, because of the presence of large size precipitated phase particles in the alloy tissue and their properties (strength, Young's modulus) are different from the solute particles', under the action of cyclic load, either the surface of the precipitated phase particles and the solute tissue appear to slide and separation, or the precipitated phase particles are brittle and fractured themselves, then the cracks initialize on the weak point of the key regions, lastly, the cracks appear. Some micro cracks merge into a large crack, and which propagate from the crack initiation region to material internal along the direction of perpendicular load, then the fatigue crack propagation region is formed and the radial pattern "stair" is left (Fig. 4 (b)). Finally, the fatigue crack transient beak region is formed while the material unable to bear the external load and fracture.
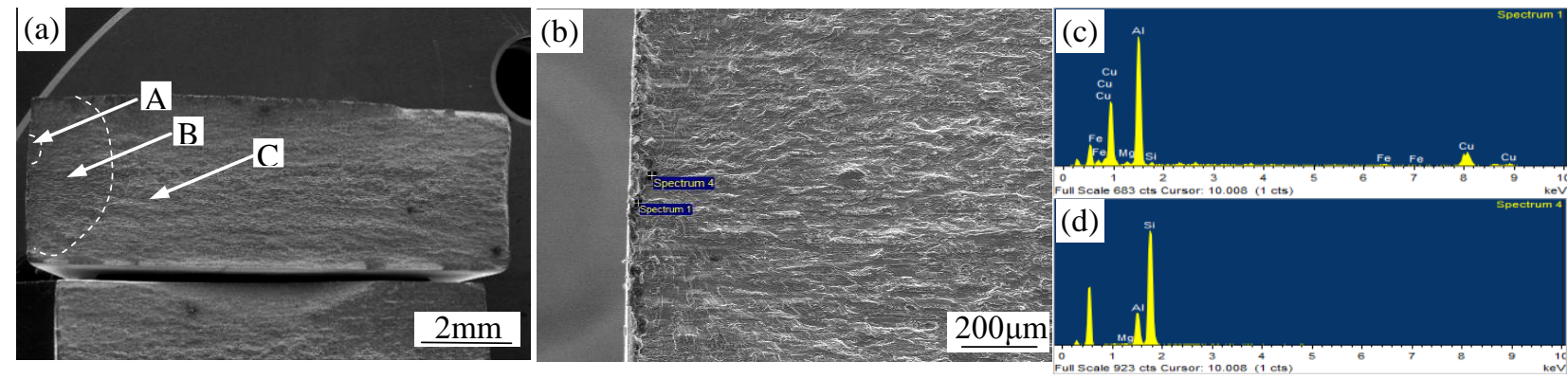

Fig. 4 Fatigue fracture morphology of $\sigma_{\max }=260 \mathrm{MPa}$ from macroscopic scale: (a) the panorama of the fracture morphology; (b) the fatigue crack initiation morphology; (c), (d): the EDS spectrum

The EDS spectrum of the precipitated phase particles as shown in Fig.4 (c) and Fig.4 (d), it can be seen that those precipitated phase particles are rich Fe intermetallic compounds which formed in the solidification process (Fig.4 (c)) and others are silicon-containing compounds (Fig.4 (d)). Therefore, reducing impurity in the high strength aluminum alloy in smelting process can greatly improve its fatigue performance.

\section{From the Microscopic Scale}

The fatigue fracture morphology of stress level $260 \mathrm{MPa}$ was observed by high magnification SEM, as shown in Fig.5. The fatigue crack propagation region is shown in Fig.5 (a), the fatigue crack growth region is a parallel serrated section, which is a typical shear fracture, there are plenty of spherical holes surround the shear region, these holes were formed by undissolved phase debond from the matrix under fatigue loading. The reason of this phenomenon is that there are a lot of nano 
strengthening phases in 7075 aluminum alloy (Fig. 5 (b)), which resisted the crack to propagate, the cracks are difficult to propagate, which lead to appear large deformation on the adjacent border, thus, the alloy fractured along the grain layer and the fracture appears small plane characteristics.

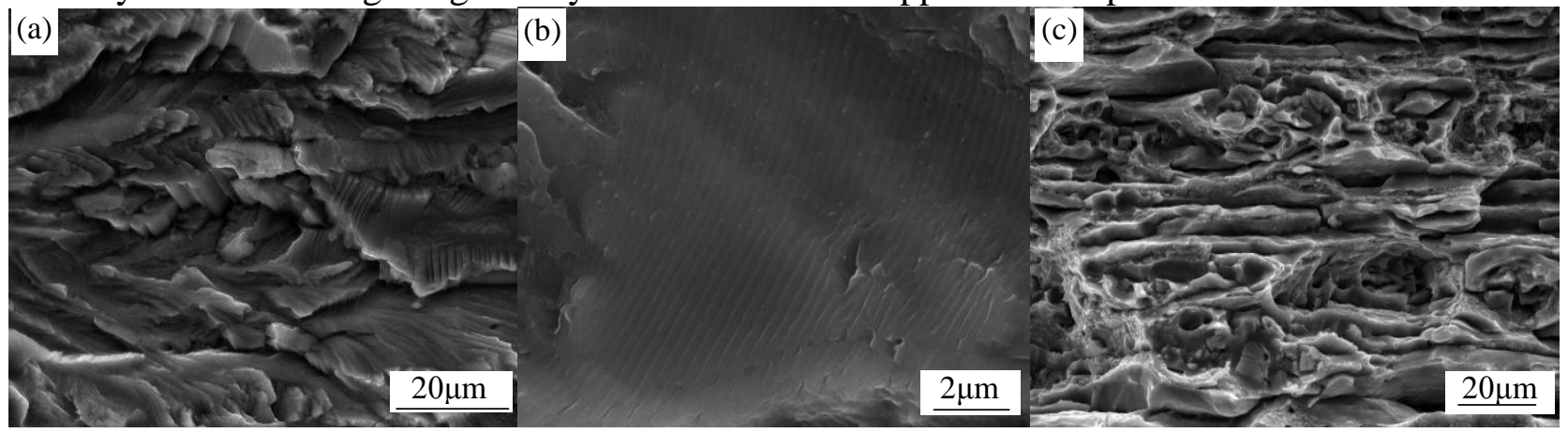

Fig. 5 Fatigue fracture morphology of $\sigma_{\max }=260 \mathrm{MPa}$ from microscopic scale:(a) fatigue crack morphology of propagation region; (b) Fatigue stripe; (c) Fatigue cracks morphology of the transient breaking region

In the crack propagation region, the fatigue stripes are clear and the stripe intervals are equal and about $0.3 \mu \mathrm{m}$, as shown in Fig. 5 (c). It reveals that the crack propagation rate is stable, in addition, there are many secondary cracks in the crack propagation region, which are propagated from the surface to the internal and parallel to the fatigue stripes. There are both intergranular fracture and transgranular fracture, in this investigation, the latter is mainly. The secondary cracks depth is much greater than the depth of the fatigue stripes.

When the fatigue crack propagated to the critical dimension, the material become instability, fractured immediately and the transient break region is formed. The transient break region has the mixed-rupture characteristics of quasi-cleavage and dimples. The surface of the fracture has a large number of torn edges and there are small equiaxial dimples on edge.

\section{Conclusions}

(1) The stress level has great influence on the fatigue life of the 7075-T651 aluminum alloy sheet. The fatigue life of aluminum alloy 7075-T651 decreases with increases of the stress amplitude and the S-N curve without horizontal section.

(2) The method can effectively improve the accuracy of the S-N curve, which provide a reference method for S-N curve fitting. Fatigue limit was obtained by this method under test conditions and the fatigue limit is149.0033MPa.

(3) The fatigue fracture macro observation revealed that the high-cycle fatigue fracture morphology of 7075-T651 aluminum alloy sheet show ductile fracture features. The main reason of the crack nucleation is that the second phase particles on the sub-surface, but the small and dispersed precipitates have a positive impact on the alloy fatigue performances. The morphology of the fatigue crack propagation is "stair" distribution. The fatigue crack transient beak region is characterized by the mixed-rupture characteristics of quasi-cleavage and dimples.

\section{Acknowledgement}

This project is supported by the National Natural Science Foundation of China (51375500); Hunan Provincial Education Department Funded Projects (2013SK2001); The Fundamental Research Funds for the Central Universities of Central South University (2013zzts037), are gratefully acknowledged.

\section{References}

[1]Z. T. Wang, R .Z. Tian, Aluminum Processing Handbook, Central South University Press, Changsha, 1988. 
[2]B. Liu, C. Q. Peng, R. C. Wang, et al., Recent development and prospects for giant plane aluminum alloys, The Chinese Journal of Nonferrous Metals. 9(2010) 1705-1715.

[3]E. Cirik, K. Genel, Effect of anodic oxidation on fatigue performance of 7075-T6 alloy, Surface \& Coatings Technology. 21 (2008) 5190-5201.

[4]T. W. Zhao, J. X. Zhang, Y. Y. Jiang, A study of fatigue crack growth of 7075-T651 aluminum alloy, International Journal of Fatigue. 30 (2008) 1169-1180.

[5]K. Walker, The effect of stress ratio during crack propagation and fatigue for 2024-T3 and 7075-T6 aluminum, Effects of environment and complex load history on fatigue life, ASTM STP. 462(1970) 1-14.

[6]R. S. Sachin, W. H. David, Fretting fatigue behavior in 7075-T6 aluminum alloy, Wear 261. (2006) 426-434.

[7]J. Han, Q. X. Dai, Y. T. Zhao, et al., Study on Fatigue Performance of 7075 -T651 Aluminum Alloys, Journal of Aeronautical Materials.4 (2010): 92-96. 